\title{
Post Tooth Extraction Necrotising Fasciitis and Rhinocerebral Mucormycosis in a Diabetic Patient
}

\author{
Ekta Gupta ${ }^{1}$, Usha Verma ${ }^{2 *}$, Prameshwar Lal ${ }^{3}$, P.C. Gupta ${ }^{4}$ and Prabhu Prakash ${ }^{2}$ \\ ${ }^{1}$ Department of Orthodonics, Vyas Dental College, Jodhpur, India \\ ${ }^{2}$ Department of Microbiology, ${ }^{3}$ Department of Surgery, Dr. S.N.Medical, College, \\ Jodhpur, India \\ ${ }^{4}$ Department of Surgery, Paota Hosp. Asso. Group of Hosp., \\ Dr S.N.Medical College Jodhpur, India \\ *Corresponding author
}

\section{A B S T R A C T}

Dentists and oral surgeons perform tooth extractions. If the tooth is impacted, they cut away gum and bone tissue which cover the tooth and then, using forceps, grasp the tooth and gently rock it back and forth to loosen it from the jaw bone and ligaments

\section{Keywords}

Mucormycosis, DM-1, ICU, Cerebralmucormyc osis, Maxillary sinusitis, Necrotising fasciitis.

Article Info

Accepted:

17 June 2018

Available Online:

10 July 2018 that hold it in place. Once the tooth has been extracted, a blood clot usually forms in the socket. Sometimes, the blood clot in the socket breaks loose, exposing the socket leading to dry socket. But when this wok of tooth extraction is performed by unauthorized and unskilled persons there is breach in protocols. If patient is immunocompromised there is risk of developing opportunistic infections may be bacterial, fungal etc. Mucormycosis is an aggressive, frequently fatal invasive fungal infection that can develop in patients with a number of predisposing conditions. We report on a 18-year-old male patient with DM who developed an invasive fungal disease of the facial bones, myositis, cerebritis after tooth extraction with uncontrolled blood sugar from some quack 10 days back and was admitted with complains of tender swelling over right cheek extending up to infraorbital region along with high grade fever and altered sensorium. Patient died in ICU due to Mucormycosis. Mucormycosis is an opportunistic fungal pathogen that has the ability to cause significant morbidity and frequently mortality in the susceptible patient. An overview of this class of pathogens and the history, examination findings (clinical and radiographic), pathogenesis and medical-surgical treatment of mucormycosis is presented.

\section{Introduction}

Cerebral mycosis is caused by a group of fungi including, saprophytic fungi, Zygomycetes group of fungi, dimorphic fungi, yeast and yeasts-like fungi. Zygomycosis is an opportunistic fungal infection caused by class Zygomycetes such as the Genera Rhizopus, Rhizomucor, Absidia, Mucor etc. and may present clinically as cutaneous, subcutaneous, 
systemic and rhinocerebral infections. Cerebral mucormycosis is becoming more frequently recognized as secondary occurrence in diabetes mellitus patients. Zygomycetes thrive in a highly acid condition that has rich carbohydrate; therefore a diabetic ketoacidosis person has a more risk of quick invasion. Burn patients or patients receiving cancer chemotherapy are also at risk (1-4).

Cases are commonly observed whenever there is any breach in aseptic precautions; commonly seen post brain surgeries or tooth extraction.

Cerebral mucormycosis is becoming more frequently recognized as secondary occurrence in diabetes mellitus, burn or patients receiving cancer chemotherapy. Zygomycetes thrive in a highly acid condition that has rich carbohydrate; therefore a diabetic ketoacidosis person has a more risk of quick invasion. Cases are commonly observed whenever there is any breach in aseptic precautions; commonly seen post brain surgeries or tooth.

Invasive fungal infections (mycoses) are uncommon, but when they occur, they are devastating to patients. These infections are opportunistic - they occur when organisms to which we are frequently exposed gain entry to the body due to a decrease in host defenses or through an invasive portal, such as a dental extraction(5-8).

Mucormycosis is a saprophytic aerobic fungus commonly found in our environment, for example, in bread moulds or decaying vegetation. This organism is frequently found to colonize the oral mucosa, nasal mucosa, paranasal sinuses and pharyngeal mucosa of asymptomatic patients. These fungi do not usually cause disease in healthy people with intact immune systems, but patients with a number of conditions can be predisposed to the development of invasive fungal disease. These conditions include diabetes mellitus, renal failure, malignancies, intravenous drug abuse, malnutrition states, as well as immunesuppression and corticosteroid therapy.

We are reporting a case of cerebral mucormycosis after tooth extraction in a young patient having uncontrolled blood sugar (DM-1) by a road sidequack.

\section{Case Report}

18years, male, admitted with complains of tender swelling over right cheek extending up to infraorbital region along with high grade fever, he gave history of tooth extraction by a quack in a state of uncontrolled blood sugar (pt. Was known case of DM-1 since last 2 years), 2 days after admission patient had altered sensorium (Glasgow Coma Scale (GCS) of patient was $\mathrm{E}_{3} \mathrm{~V}_{4} \mathrm{M}_{5}$ and B.P. was 90/60 $\mathrm{mmHg}$ sugar $>450 \mathrm{mg} / \mathrm{dl}$, urinary ketone bodies: 4+ and was hypokalaemia. After 1 day of admission, patient became unconscious with gasping respiration and so was intubated and shifted on mechanical ventilation. Meanwhile patient developed necrosis of right half of face extending to temporal region. Hypokalaemia persisted throughout the hospital course and shortness of breath and swelling extended up to right side of whole face and converted into necrotising fasciitis in spite of broad spectrum antibiotic coverage .Patient was HIV, $\mathrm{HBs} \mathrm{Ag}$ negative, blood culture was negative, tissue debribment over face was done and sent for HPE \& CULTURE. On HPE there was presence of thick, broad fungal mycelium on SDA culture it confirmed as case of Mucormycosis. In spite of Fluconazole, Voraconazole, Tygecycline, Vancomycin, Metronidazole, Mannitol and Dexona infusions patient succumbed to death four days after admission (Fig. 1-7).

MRI-Brain - cerebral mucormycosis, Maxillary sinusitis and necrotising fasciitis. 


\section{Results and Discussion}

Although permanent teeth can last a lifetime, teeth that have become damaged or decayed may need to be removed or extracted. Other reasons include crowded mouth, Gum disease or infection. Sometimes dentists extract teeth to prepare the mouth for orthodontics. The goal of orthodontics is to properly align the teeth, which may not be possible if teeth are too big for mouth. If tooth decay or damage extends to the pulp -- the center of the tooth containing nerves and blood vessels -- bacteria in the mouth can enter the pulp, leading to infection. If infection is so severe that antibiotics do not cure it, extraction may be needed to prevent the spread of infection. If immune system is compromised (for example, if patient is receiving chemotherapy or are having an organ transplant) even the risk of infection in a particular tooth may be reason to remove the tooth. If periodontal disease -- an infection of the tissues and bones that surround and support the teeth -- have caused loosening of the teeth, it may be necessary to extract the tooth or teeth. Cerebral mucormycosis is becoming more frequently recognized as secondary occurrence in diabetes mellitus, burn or patients receiving cancer chemotherapy. Zygomycetes thrive in a highly acid condition that has rich carbohydrate; therefore a diabetic ketoacidosis person has a more risk of quick invasion. Cases are commonly observed whenever there is any breach in aseptic precautions; commonly seen post brain surgeries or tooth

Invasive fungal infections (mycoses) are uncommon, but when they occur, they are devastating to patients. These infections areo pportunistic - they occur when organisms to which we are frequently exposed gain entry to the body due to a decrease in host defenses or through an invasive portal, such as a dental extraction.
Mucormycosis is a fungal infection due to organisms in the order Mucorales. These organisms belong to the general class zygomycetes, which are ubiquitous. They can be found in decaying vegetation, soil and decaying food (for example bread mould). These organisms can occasionally be cultured from non-infected individuals. This organism is frequently found to colonize the oral mucosa, nasal mucosa, paranasal sinuses and pharyngeal mucosa of asymptomatic patients. These fungi do not usually cause disease in healthy people with intact immune systems, but patients with a number of conditions can be predisposed to the development of invasive fungal disease. These conditions include diabetes mellitus, renal failure, malignancies, intravenous drug abuse, malnutrition states, as well as immune- suppression and corticosteroid therapy. Mucormycosis can have multiple clinical presentations. The most common is the rhinocerebral form, involving the nose, paranasal sinuses, orbits and central nervous system. Others include cutaneous, gastroin-testinal, pulmonary and disseminated forms. The rhinocerebral form has been sub classified into rhino maxillary and rhinocerebralorbital by some.

Rhinocerebral mucormycosis typically begins with hyphal invasion of the paranasal sinuses or the oronasal cavity of a susceptible host. Early symptoms may include paranasal paresthesias, cellulitis, periorbital edema, rhinorrhea and nasal crusting. These features are quickly superseded by eschar formation and necrosis of the naso-facial region (6).

Advancing infection can quickly result in cavernous sinus thrombosis, carotid artery or jugular vein thrombosis (Lemierre Syndrome) and death. Mucormycosis is an aggressive, frequently fatal invasive fungal infection that can develop in patients with a number of predisposing conditions. 


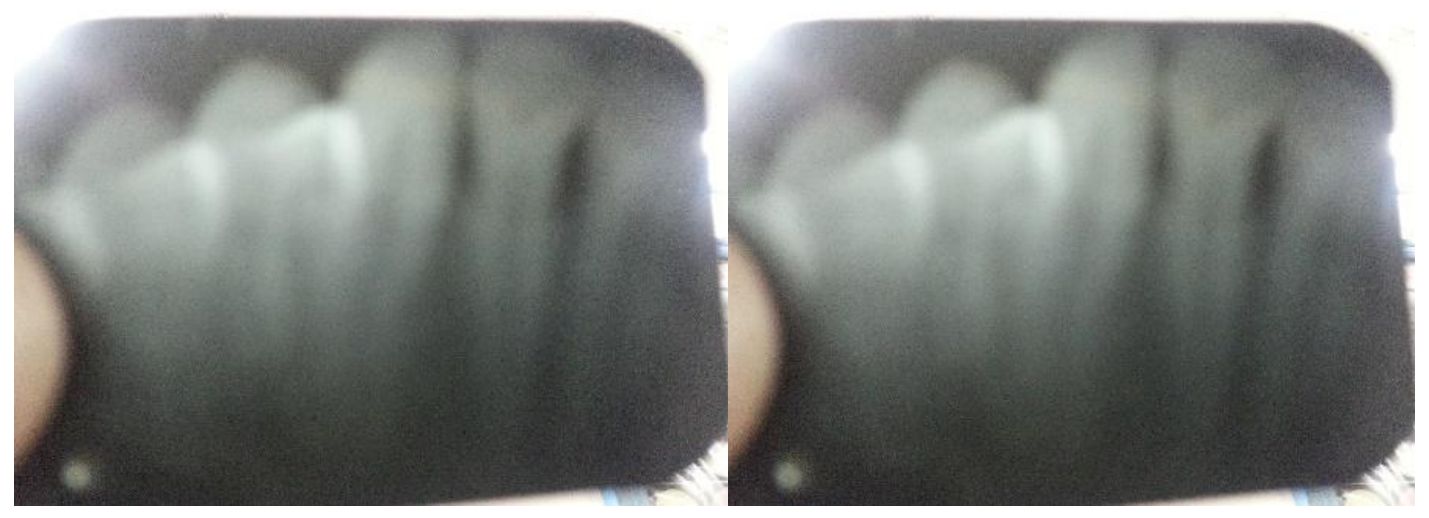

Fig. 1,2- Dental I.O.P.A. of patient

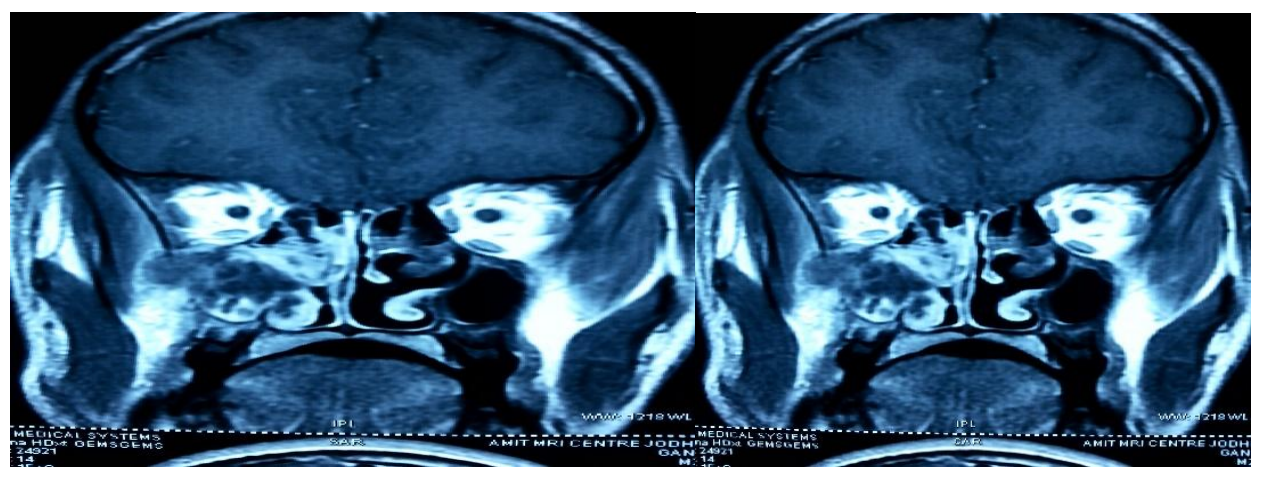

Fig. 3,4- CT scan of patient showing cerebritis

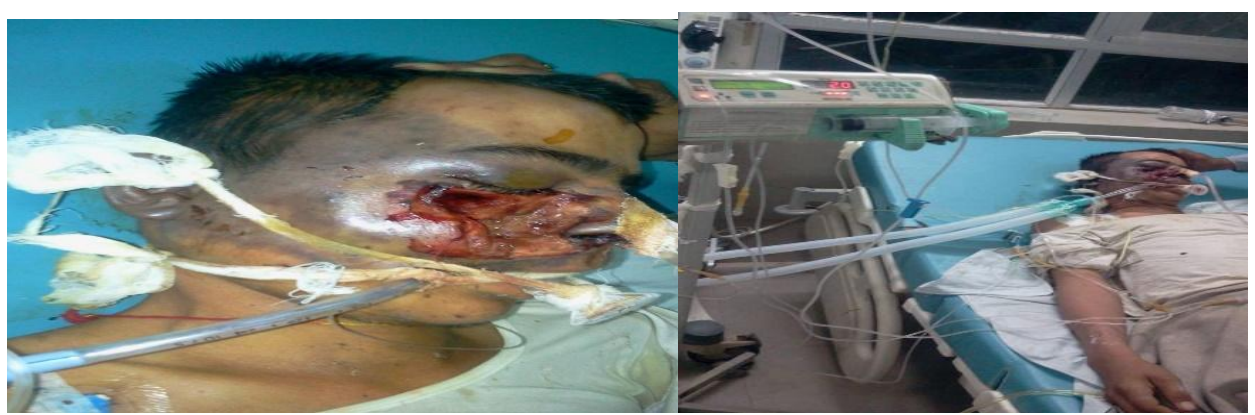

Fig. 5,6- showing fascitis and ulcer present on face over maxillary sinus

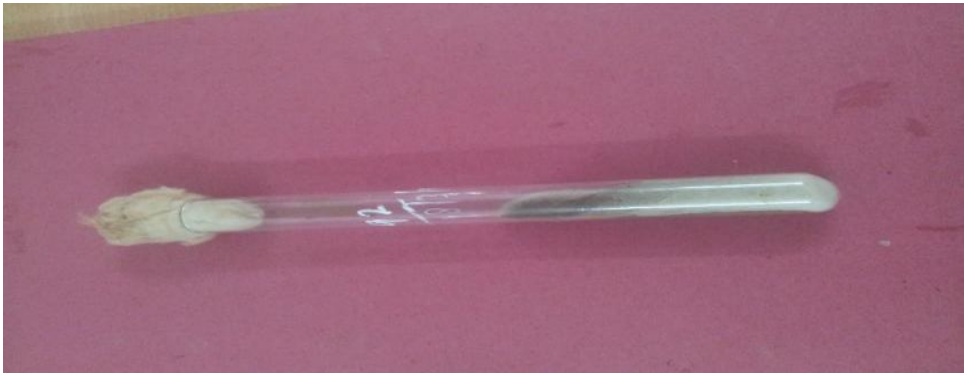

Fig.7 Showing mucor grown on sabourad dextrose agar 
In the susceptible patient, it can be triggered by minor surgical wounds, such as dental extractions. Expeditious diagnosis, systemic amphotericin B therapy, aggressive surgical debridement and optimal medical management are critical for patient survival. This case reinforces the concept that simple procedures such as dental extractions can cause catastrophic complications in susceptible patients. All dental professionals will encounter patients whose general health is tenuous and easily perturbed. Knowledge of potentially devastating complications can help to prevent the unfortunate consequences described here. In this case, tooth extraction was done by a quack in unsterilized condition with the associated DM-1 with uncontrolled Blood sugar. Preoperative check-up was not done, intraoperative sterilization and post-operative care was also compromised. Patient was ignorant about the complications. Here poor hygiene and uncontrolled blood sugar level of patient played pivotal role in invasion of fungus. Patient died due to invasion of fungus which resulted in cutaneous necrotising fasciitis and later on rhino cerebral mucormycosis. Despite of all efforts patient succumbed to death.

Patient was diagnosed as a case of diabetic ketoacidosis with rhinocerebral and subcutaneous mucormycosis which with time progressed to septicaemia with septic shock leading to respiratory failure. Patient was managed under intensive care unit with life support, vasopressor administration, i.v. antibiotics, i.v. antifungals, insulin infusion and potassium supplementation. But despite the entire vigorous measures patient died.

\section{References}

1. Toranto AM, Dho G, Pritano A, Breda G,
Grancini A. Invasive fungal infection in the ICU: a multicentric, prospective, observational study in Italy. Mycosis., 2011; 55:73-79.

2. Shmuel S, Stuart ML. The immune response to fungal infections. Brit J Haemtol., 2005, 129:569-582.

3. Monnet G, Venet F, Kullberg BJ, Mihi GN. ICU acquired immunosuppression and the risk for secondary fungal infections. Med Mycol., 2011:49:517-523.

4. Kofteridis DP, Karabekios S, Panagiotides JG, Bizakis J, Kyrmizakis D, Saridaki Z: Successful treatment of rhinocerebral mucormycosis with liposomal amphotericin B and surgery in two diabetic patients with renal dysfunction. J Chemother., 2003; 15(3):282-6.

5. Bhansali A, Bhadada S, Sharma A, Suresh V, Gupta A, Singh P, and others. Presentation and outcome of rhino-orbital-cerebral mucormycosis in patients with diabetes. Postgrad Med J 2004; 80(949):670-4..

6. Jones AC, Bentsen TY, Freedman PD. Mucormycosis of the oral cavity. Oral Surg Oral Med Oral Pathol., 1993; 75(4):455-60.

7. Talmi YP, Goldschmied-Reouven A, Bakon M, Barshack I, Wolf M, Horowitz Z, and others. Rhino-orbital and rhino-orbito-cerebral mucormycosis. Otolaryngol Head Neck Surg., 2002; 127(1):22-31.

8. Ferguson BJ. Mucormycosis of the nose and Para nasal sinuses. Otolaryngol Clin North Am., 2000; 33(2):349-65.

9. .Karam F, Chmel H. Rhino-orbital cerebral mucormycosis. Ear Nose Throat J., 1990; 69(3):187, 191-3.

10. Garcia-Covarrubias L, Barratt DM, Bartlett R, Van Meter K. [Treatment of mucormycosis with adjunctive hyperbaric oxygen: five cases treated at the same institution and review of the literature]. Rev Invest Clin., 2004; 56(1):51-5 [Spanish].

\section{How to cite this article:}

Ekta Gupta, Usha Verma, Prameshwar Lal, P.C.Gupta and Prabhu Prakash. 2018. Post Tooth Extraction Necrotising Fasciitis and Rhinocerebral Mucormycosis in a Diabetic Patient Int.J.Curr.Microbiol.App.Sci. 7(07): 2408-2412. doi: https://doi.org/10.20546/ijcmas.2018.707.281 